tionships between edges in natural scenes quantitatively predicts human ability to detect contours on complex backgrounds, under a range of conditions ${ }^{12}$.

Second, Weiss et al. ${ }^{1}$ used the standard utility function that corresponds to picking the interpretation with the maximum posterior probability (Fig. 1, black dot in lower middle panel). The choice of utility function should have a minor effect in their situation, but the choice can be important in some situations ${ }^{13,14}$. For example, if smaller errors have greater utility than larger errors, and if the posterior probability distribution contains a tall narrow peak and a short wide peak, then the maximum expected utility may be at the short peak rather than the tall peak. Also, the most appropriate utility function when considering biological vision is arguably one based on fitness ${ }^{15}$.

Third, as Weiss et al. ${ }^{1}$ point out, their Bayesian model does not consider certain fundamental biological constraints, such as the limited dynamic range and limited speed of neural responses. Such constraints undoubtedly arise (ultimately) from bio- physical limitations, and from the design compromises required because biological systems must perform many tasks. In supplementary material, Weiss et al. demonstrate that plausible neural constraints bring the predictions of their model into better quantitative agreement with the data.

Finally, real perceptual systems are designed through natural selection and perceptual learning, which sometimes find local optima in design space, rather than the global optimum of the Bayesian ideal observer. However, this fact does not diminish the value of the Bayesian approach; indeed, the concepts of Bayesian statistical decision theory lend themselves elegantly to rigorous formulations of natural selection ${ }^{15}$. Thus, even in the broader biological context of plasticity, learning and natural selection, the Bayesian approach may prove to be optimal.

1. Weiss, Y., Simoncelli, E. \& Adelson, E. H. Nat Neurosci. 5, 598-604 (2002).

2. Freeman, W. T. Nature 368, 542-545 (1994).

3. Knill, D. C., Kersten, D. \& Yuille, A. in Perception as Bayesian Inference (eds. Knill, D. C. \& Richards, R. W.) 1-21
(Cambridge Univ. Press, 1996).

4. Yuille, A. L. \& Bülthoff, H. H. in Perception as Bayesian Inference (eds. Knill, D. C. \& Richards, R. W.) 123-161 (Cambridge Univ. Press, 1996).

5. Kersten, D. in The New Cognitive Neurosciences. 2nd Edn. (ed. Gazzaniga, M. S.) 353-363 (MIT Press, Cambridge, Massachusetts, 1999).

6. Geisler, W. Psychol. Rev. 96, 267-314 (1989).

7. Liu, Z. \& Kersten, D. Vision Res. 38, 2507-2519 (1998).

8. Bloj, M. G., Kersten, D. \& Hurlbert, A. C. Nature 402, 877-879 (1999).

9. Saunders, J. A. \& Knill, D. C. Vision Res. 41, 3163-3183 (2001).

10. Mamassian, P., Landy, M. S. \& Maloney, L. T. in Statistical Theories of the Brain (eds. Rao, R., Olshausen, B. \& Lewicki, M.) 13-36 (MIT Press, Cambridge, Massachusetts, 2002).

11. Ernst, M. O. \& Banks, M. S. Nature 415, 429-433 (2002).

12. Geisler, W. S., Perry, J. S., Super, B. J. \& Gallogly, D. P. Vision Res. 41, 711-724 (2001).

13. Brainard, D. H. \& Freeman, W. T. J. Opt. Soc. Am. A 14, 1393-1411 (1997).

14. Schrater, P. R. \& Kersten, D. How optimal depth cue integration depends on the task. Int. J. Comput. Vision 40, 73-91 (2000).

15. Geisler, W. S. \& Diehl, R. Phil. Trans. R. Soc. Lond. B Biol. Sci. 357, 419-448 (2002).

\title{
Wnt signals lead cells down the caudal path
}

During development, progenitor cells along the rostrocaudal axis of the neural tube are instructed to become forebrain, midbrain, hindbrain or spinal cord. Cells of the caudal brain are believed to arise through reprogramming ('caudalization') of cells that initially show characteristics of rostral brain. On page 525 of this issue, Thomas Edlund and

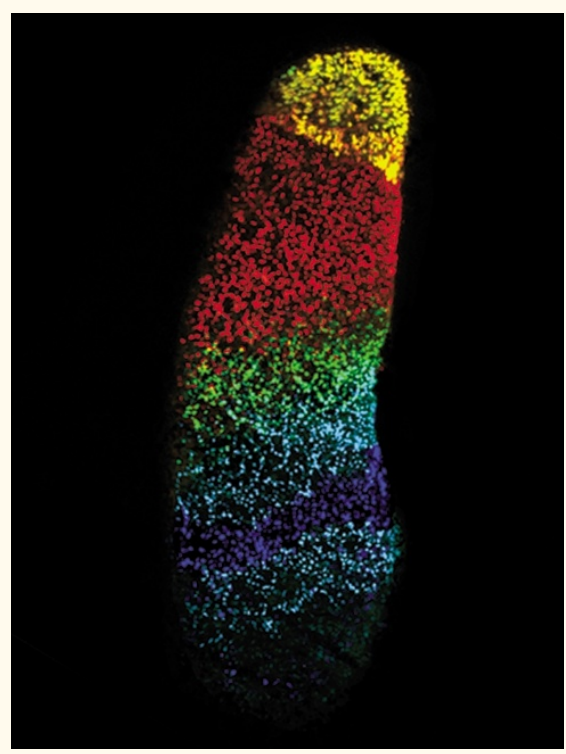
colleagues now report that Wnt signals from the posterior mesoderm are required for caudalization. FGF and retinoic acid also contribute to the induction of midbrain, hindbrain and spinal cord, but previous studies showed that these factors were not sufficient on their own. Despite earlier indications that Wnt signals were involved in specifying caudal brain character, because they have a variety of other functions in development, it was unclear whether their role in caudalization was direct or indirect. Therefore, the present work provides a crucial additional piece in this developmental puzzle.

To examine the role of Wnt signaling, the authors used explant cultures of chick neural plate along with immunohistochemical labeling for expression of a combination of transcription factors which selectivity delineate the various brain regions (yellow, rostral forebrain; red/green, rostral and caudal midbrain; light/dark blue, rostral hindbrain). When explants of caudal neural plate were taken from a stage at which the cells still exhibited primarily rostral characteristics and were cocultured with caudal mesoderm, cells expressed markers for caudal brain regions. If Wnt signaling was inhibited, however, the cells retained their rostral character. If the authors then cultured neural plate explants from a later stage when cells were already specified to eventually make rostral, middle and caudal brain regions, they found that Wnt signaling was still directly necessary for the induction of caudal character. Finally, when explants from the eventual rostral forebrain region of the neural plate were cultured in the presence of FGF and varying concentrations of Wnt conditioned medium, the authors found that increasing concentrations of Wnt resulted in expression of progressively more caudal brain markers. Therefore, the new results firmly establish a direct role for a graded Wnt signal in directing the caudalization of neural plate cells during early neural tube development.

\section{Brian Fiske}

